\title{
Precision Medicine in Alcohol Dependence: A Controlled Trial Testing Pharmacotherapy Response Among Reward and Relief Drinking Phenotypes
}

\author{
Karl Mann*,', Corey R Roos², Sabine Hoffmann', Helmut Nakovics', Tagrid Leménager', Andreas Heinz ${ }^{3}$ and \\ Katie Witkiewitz ${ }^{2}$ \\ 'Department of Addictive Behavior and Addiction Medicine, Central Institute of Mental Health, Medical Faculty Mannheim, Heidelberg University, \\ Mannheim, Germany; ${ }^{2}$ Department of Psychology, Center on Alcoholism, Substance Abuse, and Addictions, University of New Mexico, Albuquerque \\ NM, USA; ${ }^{3}$ Department of Psychiatry and Psychotherapy, Charité Universitätsmedizin Berlin, Berlin, Germany
}

\begin{abstract}
Randomized trials of medications for alcohol dependence (AD) often report no differences between active medications. Few studies in AD have tested hypotheses regarding which medication will work best for which patients (ie, precision medicine). The PREDICT study tested acamprosate and naltrexone vs placebo in 426 randomly assigned AD patients in a 3-month treatment. PREDICT proposed individuals whose drinking was driven by positive reinforcement (ie, reward drinkers) would have a better treatment response to naltrexone, whereas individuals whose drinking was driven by negative reinforcement (ie, relief drinkers) would have a better treatment response to acamprosate. The goal of the current analysis was to test this precision medicine hypothesis of the PREDICT study via analyses of subgroups. Results indicated that four phenotypes could be derived using the Inventory of Drinking Situations, a 30-item self-report questionnaire. These were high reward/high relief, high reward/low relief, low reward/high relief, and low reward/low relief phenotypes. Construct validation analyses provided strong support for the validity of these phenotypes. The subgroup of individuals who were predominantly reward drinkers and received naltrexone vs placebo had an 83\% reduction in the likelihood of any heavy drinking (large effect size). Cutoff analyses were done for clinical applicability: individuals are reward drinkers and respond to naltrexone if their reward score was higher than their relief score AND their reward score was between 12 and 31 . Using naltrexone with individuals who are predominantly reward drinkers produces significantly higher effect sizes than prescribing the medication to a more heterogeneous sample. Neuropsychopharmacology (2018) 43, 89I-899; doi:I0.1038/npp.2017.282; published online I3 December 2017
\end{abstract}

\section{INTRODUCTION}

Alcohol dependence (AD) creates enormous suffering (World Health Organization, 2009), yet the effect sizes for behavioral interventions and pharmacotherapies targeting $\mathrm{AD}$ are small to medium (van den Brink et al, 2013; Jonas et al, 2014; Magill and Ray, 2009; O'Malley et al, 1996). There are also several negative trials of $\mathrm{AD}$ treatments (Litten $e t$ al, 2013). For example, the PREDICT study (Mann et al, 2013), which examined the efficacy of acamprosate and naltrexone vs placebo in reducing heavy drinking among individuals recruited from inpatient $\mathrm{AD}$ treatment facilities, found no significant differences among conditions in the primary outcome of no heavy drinking days (success rates: $48.3 \%$ acamprosate; $49.1 \%$ naltrexone; and $51.8 \%$ placebo).

*Correspondence: Professor K Mann, Department of Addictive Behavior and Addiction Medicine, Central Institute of Mental Health, Medical Faculty Mannheim, Heidelberg University, J5, Mannheim D-68I59, Germany, Tel: +(49) 621-1703 2952, Fax: +(49) 6211703 3505, E-mail: karl.mann@zi-mannheim.de

Received 13 June 2017; revised 2 November 2017; accepted 12 November 2017; accepted article preview online 20 November 2017
One explanation for the null PREDICT study findings and other negative trials (Jonas et al, 2014) is the heterogeneity of the disorder (Litten et al, 2015). To advance the field of $\mathrm{AD}$ treatment it has been recommended that identifying subtypes of individuals with distinct genetic, neurobiological, or psychological characteristics might confer benefit of one treatment approach over a different treatment approach (ie, precision medicine; Institute of Medicine, 1990; Verheul et al, 1999).

Consistent with a precision medicine approach, the PREDICT study (Mann et al, 2009) was designed to test differential efficacy of naltrexone and acamprosate based on individual differences in neurotransmitter dysfunction associated with chronic AD neuroadaptation (Heilig et al, 2010; Koob and Volkow, 2010; Verheul et al, 1999). It was hypothesized that individuals with significant alteration of the opioidergic systems and who drink alcohol primarily in the context of positive reinforcement (reward drinkers) would benefit more from naltrexone (Heinz et al, 2003; Verheul et al, 1999). Individuals with significant glutamatergic dysfunction who primarily drink alcohol in the context of negative reinforcement (relief drinkers) may benefit more 
from acamprosate (Littleton, 1995; Spanagel and Kiefer, 2008).

The reward and relief drinking phenotypes are based on several models of addiction (Koob and Volkow, 2010; Spanagel and Weiss, 1999). For example, according to the Koob and Volkow (2010) addiction cycle, the initial stages of $\mathrm{AD}$ (binge/intoxication stage) are primarily maintained through positive reinforcement and seeking euphoria (ie, reward seeking). In later stages, alcohol is primarily consumed to avoid or reduce aversive negative affective states (ie, seeking relief) that may arise from repeated withdrawal (withdrawal/negative affect stage).

Given the prominence of reward and relief drinking tendencies in models of $\mathrm{AD}$, it is important to consider methods by which clinicians could easily identify reward and relief drinking phenotypes. Neuroimaging methods and genotyping have been proposed for detecting phenotypes for precision medicine approaches to $\mathrm{AD}$ (Heilig and Leggio, 2016; Litten et al, 2015; Spanagel et al, 2013). Neuroimaging studies have found reward circuitry engagement in response to alcohol cues (Mann et al, 2014; Schacht et al, 2013), but studies of relief drinking tendencies have been less conclusive (Hillmer et al, 2015; Sinha and Li, 2007). With respect to genotyping, there is mixed evidence that individuals with the A118G polymorphism of the OPRM1 gene may have a better treatment response with naltrexone (Kranzler et al, 2013; Oslin et al, 2015; Schacht et al, 2017). Yet, an easy to administer self-report measure could become a valuable tool for clinicians to apply precision medicine approaches in practice.

Along these lines, it is necessary to identify construct valid and easy to administer measures of reward and relief drinking. Mixture modeling is one promising analytical method for precision medicine. In an earlier analysis of the PREDICT data, Glöckner-Rist et al (2013) used mixture modeling to examine 10 items from the temptation scale of the Alcohol Abstinence Self-Efficacy Scale (AASE) and found four subgroups: low reward and relief; high reward and relief; high relief and low reward; and high reward and low relief. Roos et al (2017) identified similar subgroups using the AASE and found that relief drinkers had a significantly better treatment response to acamprosate.

\section{Current Paper}

The goal of the current paper was to conduct a hypothesisdriven subgroup analysis of the PREDICT study (Clinical Trial Registry: NCT00317031; Mann et al, 2009) to examine whether reward $v s$ relief drinking significantly moderated treatment response. Mixture modeling was used to test whether individuals could be classified into different patterns based on reward and relief drinking, and whether patterns of reward and relief drinking predicted differential treatment response to naltrexone and acamprosate. It was hypothesized that individuals with primarily reward drinking tendencies would respond better to naltrexone given its action on the opioidergic systems to reduce the rewarding effects of alcohol, whereas individuals with primarily relief drinking tendencies would respond better to acamprosate given its action on the glutamatergic systems to provide relief from negative affective states and withdrawal symptoms (Koob and Mason, 2016). Similar to Glöckner-Rist et al (2013) and

Roos et al (2017) we anticipated four different patterns of reward and relief drinking: high reward/high relief; high reward/low relief; high relief/low reward; and low reward/ low relief.

\section{MATERIALS AND METHODS}

\section{Patients and Procedures}

The PREDICT study (Mann et al, 2009, 2013) was a four-site randomized controlled trial conducted in Germany that tested the efficacy of naltrexone and acamprosate in the treatment of AD. Enrolled participants $(n=426)$ were randomly assigned to one of three treatment conditions in proportions of 2:2:1: naltrexone (50 mg daily); acamprosate ( $2 \mathrm{~g}$ daily); or placebo. Participants received pharmacotherapy over the course of 12 weeks. In addition, all patients received Medication Management, a manualized counseling approach (Pettinati et al, 2004). All participants were recruited from inpatient medical detoxification programs of 2-3 weeks' duration and were enrolled in the study upon completion of inpatient medical detoxification. Inclusion criteria included the following: diagnosis of $\mathrm{AD}$; 4-28 days of abstinence before study entry; and consumption of more than 14 drinks (for women, based on $14 \mathrm{~g}$ standard drinks $=196 \mathrm{~g}$ ) or more than 21 drinks (for men, based on $14 \mathrm{~g}$ standard drinks $=294 \mathrm{~g}$ ) per week during a consecutive 30-day period. Exclusion criteria included the following: history of other substance abuse (except nicotine and cannabis); psychiatric disorders requiring medication; and unstable medical conditions. Demographic data for the full sample: mean age $=45.3(\mathrm{SD}=8.7)$; male $(77 \%)$; married $(37 \%)$; employed (48\%); and $12+$ years of education $(51 \%)$.

\section{Measures}

Reward and relief drinking tendencies. Reward and relief drinking was measured with a 30 -item version of the Inventory of Drinking Situations (IDS; Annis et al, 1987), a self-report questionnaire that asks participants to rate the frequency $(0=$ never, $3=$ almost always $)$ of heavy drinking in various situations over the past year. Fifteen items measure reward drinking tendencies and 15 items measure relief drinking tendencies (Victorio-Estrada et al, 1996; Appendix).

Alcohol use. Alcohol consumption was measured with the Form 90 (Miller, 1996). The primary alcohol use outcomes examined in the current analysis were any heavy drinking (ie, $4+/ 5+$ standard drinks for women/men on a given drinking episode) and time to first heavy drinking episode during treatment.

Other covariates. AD severity was measured by the Alcohol Dependence Scale (Skinner and Horn, 1984). Depressive symptoms were measured with the Beck Depression Inventory-II (Beck et al, 1996). Anxiety symptoms were measured with the trait version of the State Trait Anxiety Inventory (Spielberger et al, 1983). Perceived stress was measured with the Perceived Stress Scale (Cohen et al, 1983). Harm avoidance, a trait characterized by worry, shyness, and tendency to fatigue, was measured with the Harm Avoidance subscale of the Temperament and Character Inventory (TCI; 
Cloninger et al, 1993). Novelty seeking, a trait characterized by excitability and pursuit of novel/rewarding cues, impulsivity, and loss of temper, was measured with the Novelty Seeking subscale of the TCI. Smoking status was measured at baseline with the Fagerström Test for Nicotine Dependence (Heatherton, et al, 1991).

Genotyping. Patients were genotyped to test for the OPRM1 gene polymorphism (Treutlein et al, 2009).

\section{Statistical Analyses}

Mplus Version 7.4 (Muthén and Muthén, 2012) was used to conduct all latent variable models. Missing data modeling using maximum likelihood estimation with robust standard errors was used to estimate the latent variable models and thus all available data were included in the analyses. SPSS Version 23 was used to conduct all other analyses.

Confirmatory factor analyses. We used confirmatory factor analyses (CFA) to test a latent variable model in which a reward drinking factor predicted the 15 reward items and a relief drinking factor predicted the 15 relief items (Supplementary Table 2). Model fit was evaluated by $\chi^{2}$, the root mean square error of approximation (RMSEA $<0.09$ ), and the comparative fit index (CFI $>0.97)$.

Factor mixture models. We utilized factor mixture models (FMM) to identify subgroups of individuals with distinct patterns of reward and relief drinking. FMM can be used to empirically derive subgroups of individuals who are similar with respect to underlying factor scores. We considered several indices when choosing the optimal FMM solution (Nylund et al, 2007), as well as the parsimony and theoretical utility. Most likely subgroup assignment from the final solution was saved using the estimated posterior probabilities of subgroup membership.

Construct validity analyses. We assessed the construct validity of subgroups by testing differences among the classes in gender, age, baseline days abstinent, AD severity, depressive symptoms, anxiety, perceived stress, age of onset of $\mathrm{AD}$, harm avoidance, novelty seeking, genotype, and smoking status. We utilized one-way ANOVA and Tukey's HSD post hoc test.

Main effect of latent subgroups on drinking outcomes. We tested the main effect of subgroups using logistic regression for models predicting any heavy drinking and Cox proportional hazard regression models for predicting time to first heavy drinking episode.

Interaction between latent subgroups and treatment response analyses. We tested whether subgroup interacted with medication assignment in the prediction of any heavy drinking and time to first heavy drinking episode during treatment. Dummy-coded variables of subgroup assignment were created for the analyses. We also created dummy-coded variables for medication assignment $(1=$ active medication, $0=$ placebo). Next, we created interaction terms by multiplying subgroup with the medication assignment. For each interaction model, we included the interaction terms, subgroup assignment, medication assignment, and several covariates as predictors of each of drinking outcome. Significant omnibus interaction effects $(p<0.05)$ were probed in subsequent simple slopes analyses.

In the main effects models and the interaction models, we included gender, age, $\mathrm{AD}$ severity, days abstinent before randomization, and smoking status as covariates. In addition, we ran sensitivity analyses by conducting the interaction models among participants who demonstrated medication compliance by adhering to at least $80 \%$ of their medication regimen (based on pill count, Mann et al, 2013) during treatment, and by controlling for additional covariates that were significantly different among the latent classes.

\section{Clinical Application: Creating Subgroups Using Cutoffs of Observed IDS Scores}

To increase the clinical utility of the subgroup analyses, we conducted supplementary analyses in which we identified reward drinkers in the sample based on observed scores for the IDS-30 reward and relief subscales (Appendix). The cutoffs were then used to create a binary 'reward drinker' moderator variable that was tested in interactional models.

\section{RESULTS}

\section{Confirmatory Factor Analysis}

A two-factor CFA, with reward and relief drinking factors, provided an adequate fit to the data $\left(\chi^{2}(404)=1053.49\right.$, $p<0.001$; RMSEA $=0.061$; CFI $=0.978)$. The internal consistency reliability was excellent $(\alpha=0.96)$ for both factors. The correlation of the reward and relief factor was significant $(r=0.409, p<0.001)$.

\section{Factor Mixture Model}

We chose a four-class FMM based on the collection of fit statistics supporting a four-class solution (Supplementary Table 1), as well as the meaningful pattern of item response probabilities in the four-class solution (Supplementary Table 2 ) that demonstrated patterns of reward and relief drinking that were most closely linked to substantive theory (Verheul et al, 1999). Item response probabilities were used to provide labels for each of the four latent classes, and latent class prevalences were examined to determine the proportion of the sample expected to be classified within each subgroup. Class 1, the 'all high subgroup,' was characterized by high frequency of endorsing both relief and reward drinking (prevalence $=0.28, n \approx 119$ ). Class 2, the 'reward subgroup,' was characterized by high frequency of reward drinking and low frequency of relief drinking (prevalence $=0.26, n \approx 111$ ). Class 3, the 'relief subgroup,' was characterized by high frequency of relief drinking and low frequency of reward drinking (prevalence $=0.27, n \approx 117$ ). Class 4 , the 'all low subgroup,' was characterized by low frequency of both relief and reward drinking (prevalence $=0.19, n \approx 79$ ).

We also examined differences on the observed IDS reward and relief subscale scores (Table 1). The all high subgroup had significantly higher reward and relief scores, the reward subgroup had significantly higher reward scores than the 
Table I Comparisons among Latent Classes (ie, Subgroups) on Observed IDS Subscale Scores and Covariates

\begin{tabular}{|c|c|c|c|c|c|c|c|c|c|c|c|}
\hline & \multicolumn{4}{|c|}{ Means (SD) or N (\%) } & \multirow[b]{2}{*}{$\begin{array}{l}\text { Overall } \\
\text { test }\end{array}$} & \multicolumn{6}{|c|}{ Class comparisons } \\
\hline & $\begin{array}{l}\text { Class I: } \\
\text { all high } \\
(n=I 19)\end{array}$ & $\begin{array}{l}\text { Class 2: } \\
\text { reward } \\
(n=I I I)\end{array}$ & $\begin{array}{l}\text { Class 3: } \\
\text { relief } \\
(n=I I 7)\end{array}$ & $\begin{array}{l}\text { Class 4: } \\
\text { all low } \\
(n=79)\end{array}$ & & I vs 2 & I vs 3 & I vs 4 & 2 vs 3 & 2 vs 4 & 3 vs 4 \\
\hline \multicolumn{12}{|l|}{ Observed IDS subscales } \\
\hline Reward Subscale & $28.13(6.79)$ & $21.50(6.48)$ & $9.24(5.27)$ & $3.49(3.47)$ & *** & *** & *** & *** & *** & *** & **⿻丷木 \\
\hline \multicolumn{12}{|l|}{ Covariates } \\
\hline Male gender & $97(81.5 \%)$ & $93(83.8 \%)$ & $81(69.2 \%)$ & $56(70.9 \%)$ & $*$ & NS & *** & *** & 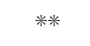 & 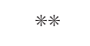 & NS \\
\hline Age & $42.71(8.22)$ & $45.54(10.03)$ & $45.31(7.75)$ & $48.75(7.45)$ & **⿻丷木 & $*$ & $*$ & **⿻丷木 & NS & NS & NS \\
\hline Baseline days abstinent & $22.15(4.10)$ & $21.95(4.20)$ & $22.25(4.40)$ & $22.14(4.40)$ & NS & & & & & & \\
\hline PSS & $19.18(7.02)$ & $12.79(5.63)$ & $17.28(6.56)$ & $12.08(6.47)$ & **⿻丷木 & **⿻丷木 & NS & *** & *** & NS & $* *$ \\
\hline Age of onset of AUD & $28.01(8.74)$ & $30.59(10.25)$ & $32.51(10.25)$ & $33.27($ ( I .09) & *** & NS & *** & *** & NS & NS & NS \\
\hline TCl-harm avoidance & $19.37(7.28)$ & $15.16(5.18)$ & $17.86(5.99)$ & $14.12(5.53)$ & *** & *** & NS & **⿻丷木 & **⿻丷木 & NS & *** \\
\hline TCl-novelty seeking & $21.13(5.30)$ & $20.38(4.82)$ & | $8.42(5.55)$ & $19.35(5.27)$ & 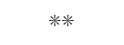 & NS & **⿻丷木 & NS & $*$ & NS & NS \\
\hline G-allele genotype & $24(23 \%)$ & $15(15 \%)$ & 25 (23\%) & 20 (29\%) & NS & & & & & & \\
\hline Smoker & 91 (77\%) & 86 (78\%) & $89(76 \%)$ & 49 (62\%) & NS & & & & & & \\
\hline
\end{tabular}

Abbreviations: ADS, Alcohol Dependence Severity Scale; AUD, alcohol use disorder; BDI, Beck Depression Inventory; PSS, Perceived Stress Scale; STAI, State-Trait Anxiety Inventory; TCl, Temperament and Character Inventory.

* $p<0.05$, **** $p<0.01$

Follow-up class comparisons were only examined if the overall test was statistically significant. For continuous variables, ANOVA and Tukey's HSD post hoc test were conducted. For categorical variables, $\chi^{2}$-tests were conducted.

relief and all low subgroups, and the relief subgroup had significantly higher relief scores than the reward and the all low subgroups.

\section{Construct Validity Analyses: Clinical and Genotyping Results}

The construct validity analyses revealed statistically significant, theoretically consistent, and clinically meaningful differences among the four subgroups on relevant covariates (Table 1). The reward subgroup exhibited relatively high novelty seeking, low harm avoidance, moderate dependence severity, and low depressive symptoms, perceived stress, and anxiety, as well as a higher proportion of men. The relief class exhibited higher harm avoidance, low novelty seeking, high dependence severity, and moderate to high scores for depressive symptoms, perceived stress, anxiety, and harm avoidance. There were no significant differences among the four subgroups in baseline days abstinent, OPRM1 G-allele genotype, current age, age of onset of $\mathrm{AD}$, and smoking status (Table 1).

\section{Main Effect of Latent Class on Drinking Outcomes with Low Class as Reference Class}

There were no main effects of reward and relief drinking subgroup on drinking outcomes. As compared to the all low subgroup, the all high subgroup did not significantly predict any heavy drinking or the time to first heavy drinking episode during treatment $(p>0.34)$ and the reward subgroup did not significantly predict any heavy drinking or time to first heavy drinking episode during treatment $(p>0.86)$. The relief subgroup did not significantly predict any heavy drinking or time to first heavy drinking episode during treatment $(p>0.24)$.

\section{Interaction Between Classes and Medication Response}

Consistent with our hypothesis, there was a significant interaction between the reward subgroup (with the all low subgroup as the reference group) and naltrexone in the prediction of any heavy drinking during treatment (Table 2). However, when time to first heavy drinking episode was the end point the interaction between reward subgroup and naltrexone was not significant $(B \quad(\mathrm{SE})=-1.30 \quad(0.67)$, $p=0.053$ ). Simple slope models (Table 3 ) indicated that in the reward subgroup, naltrexone (relative to placebo) significantly predicted an $83 \%$ decreased likelihood of any heavy drinking $(B(\mathrm{SE})=-1.77(0.69), \mathrm{OR}=0.17, p=0.010$, corresponding to a large effect size), indicating a positive treatment response to naltrexone in the reward subgroup. In the all low subgroup, naltrexone (relative to placebo) did not significantly predict any heavy drinking, indicating that naltrexone conferred no significant benefit over placebo for those in the all low subgroup. 
Counter to our hypothesis there was also a significant interaction between the reward subgroup (all low subgroup as reference group) and acamprosate in the prediction of any heavy drinking during treatment. However, simple slope

Table 2 Summary of Results Across Models of Subgroup $\times$ Treatment Condition Predicting Drinking Outcomes During Treatment With All Low Subgroup as Reference Group

\begin{tabular}{lccc}
\hline & $\begin{array}{c}\text { Any heavy } \\
\text { drinking }\end{array}$ & & $\begin{array}{c}\text { Time to first heavy } \\
\text { drinking day }\end{array}$ \\
\cline { 2 - 2 } Predictor & B (SE) & & B (SE) \\
\hline All high $\times$ acamprosate & $-0.57(0.84)$ & & $-0.44(0.63)$ \\
Reward $\times$ acamprosate & $-1.92(0.92)^{*}$ & & $-1.17(0.63)$ \\
Relief $\times$ acamprosate & $0.26(0.82)$ & & $0.19(0.63)$ \\
All high $\times$ naltrexone & $0.29(0.86)$ & & $0.04(0.65)$ \\
Reward $\times$ naltrexone & $-1.96(0.95)^{*}$ & & $-1.30(0.67)$ \\
Relief $\times$ naltrexone & $0.72(0.85)$ & & $0.33(0.66)$ \\
\hline
\end{tabular}

* $p<0.05$.

Logistic regression was used for models predicting any heavy drinking and Cox

proportional hazard regression for models predicting time to first heavy drinking episode. analyses revealed this interaction was nonsignificant in the simple slopes model and largely driven by poor response to acamprosate among individuals in the all low subgroup instead of a better treatment response in the reward class.

These results were further tested by analyzing the subsample of medication compliers (at least an $80 \%$ adherence rate; $n=294$ ) and by examining the effects of additional covariates (in addition to gender, age, severity, days abstinent, and smoking status). Among medication compliers, the interaction between naltrexone and the reward subgroup significantly predicted any heavy drinking $(p=0.03)$ and the interaction between acamprosate and the reward subgroup was not significant in the prediction of any heavy drinking $(p=0.11)$. Similarly, controlling for additional covariates, including depressive symptoms, trait anxiety, perceived stress, age of $\mathrm{AD}$ onset, harm avoidance, and novelty seeking, led to the same substantive pattern of results: there was a significant naltrexone-by-reward class interaction in the prediction of any heavy drinking $(p=0.04)$ and the acamprosate-by-reward class interaction was not significant $(p=0.07)$.

Table 3 Summary of Medication Effects within Latent Class

Any heavy drinking during treatment as outcome

Naltrexone effect B (SE)

Acamprosate effect B (SE)

n (\%) with any heavy drinking

\begin{tabular}{lcc}
\hline All high subgroup & $0.44(0.55)$ & $0.1 \mathrm{I}(0.55)$ \\
& $\mathrm{OR}=1.55$ & $\mathrm{OR}=1.1 \mathrm{I}$ \\
& $-1.77(0.69)^{*}$ & $-1.31(0.67)$ \\
Reward subgroup & $\mathrm{OR}=0.17$ & $\mathrm{OR}=0.27$ \\
& & \\
Relief subgroup & $0.86(0.52)$ & $0.89(0.5 \mathrm{I})$ \\
& $\mathrm{OR}=2.36$ & $\mathrm{OR}=2.44$ \\
& & \\
All low subgroup & $0.23(0.71)$ & $0.66(0.68)$ \\
& $\mathrm{OR}=1.26$ & $\mathrm{OR}=1.94$
\end{tabular}

Naltrexone group: 3I (62.0\%)

Acamprosate group: 23 (51.1\%)

Placebo group: 12 (50.0\%)

Naltrexone group: 17 (36.2\%)

Acamprosate group: 22 (43.1\%)

Placebo group: 12 (75.0\%)

Naltrexone group: 26 (59.1\%)

Acamprosate group: 28 (60.9\%)

Placebo group: II (40.7\%)

Naltrexone group: 12 (42.9\%)

Acamprosate group: 16 (48.5\%)

Placebo group: 6 (33.3\%)

\begin{tabular}{|c|c|c|c|}
\hline \multicolumn{4}{|c|}{ Time (in days) to first heavy drinking during treatment as outcome } \\
\hline & Naltrexone effect $B$ (SE) & Acamprosate effect $B(\mathrm{SE})$ & Mean (SD) \\
\hline All high subgroup & $\begin{array}{l}0.10(0.36) \\
O R=1.10\end{array}$ & $\begin{array}{c}-0.03(0.37) \\
O R=0.97\end{array}$ & $\begin{array}{l}\text { Naltrexone group: } 53.80(33.13) \\
\text { Acamprosate group: } 58.87 \text { (35.07) } \\
\text { Placebo group: } 59.17 \text { (36.88) }\end{array}$ \\
\hline Reward subgroup & $\begin{array}{c}-1.09(0.40)^{* * *} \\
\mathrm{OR}=0.34\end{array}$ & $\begin{array}{l}-0.7 \mathrm{I}(0.36) \\
\mathrm{OR}=0.49\end{array}$ & $\begin{array}{l}\text { Naltrexone group: } 71.72(28.00) \\
\text { Acamprosate group: } 62.13(33.67) \\
\text { Placebo group: } 46.37(37.80)\end{array}$ \\
\hline All low subgroup & $\begin{array}{l}0.04(0.56) \\
O R=1.05\end{array}$ & $\begin{array}{l}0.36(0.54) \\
O R=1.43\end{array}$ & $\begin{array}{l}\text { Naltrexone group: } 68.39(34.01) \\
\text { Acamprosate group: } 61.76(33.16) \\
\text { Placebo group: } 68.39 \text { (34.0I) }\end{array}$ \\
\hline
\end{tabular}

Abbreviation: $O R$, odds ratio.

${ }^{*} p<0.05, * * p<0.01$.

Logistic regression was used for models predicting any heavy drinking and Cox proportional hazard regression for models predicting time to first heavy drinking episode. 


\section{Clinical Application: Creating Subgroups Using Cutoffs of Observed IDS Scores}

When using the cutoff criteria (Appendix) of the reward subscale score being higher than the relief subscale score and the reward subscale score being between 12 and 31 to identify reward drinkers $(n=148 ; 35 \%)$, there was a significant overall interaction effect between the reward subgroup and naltrexone in the prediction of any heavy drinking $(p<0.05)$ and a large effect of naltrexone vs placebo among reward drinkers $(\mathrm{OR}=0.22)$.

\section{DISCUSSION}

Clinical criteria for the diagnosis of $\mathrm{AD}$ are diverse and result in heterogeneity in the clinical presentation of patients (Institute of Medicine, 1990). Precision medicine tries to define more homogeneous subgroups of patients and match them with specifically tailored treatment (Kranzler and McKay, 2012). On the basis of specific hypotheses (Clinical Trials registry NCT00317031), the PREDICT study was designed to test the basic assumptions of precision medicine in the treatment of $\mathrm{AD}$ patients. We had the first objective of examining the effects of naltrexone and acamprosate in the full sample (as reported in Mann et al, 2013) and also proposed a second objective to examine whether subgroups of patients would respond differentially to naltrexone and acamprosate (Mann et al, 2009): 'reward drinkers' would benefit from naltrexone, whereas 'relief drinkers' would benefit from acamprosate.

The current subgroup analyses of the PREDICT data tested the second objective of the PREDICT study to examine the $a$ priori hypothesis regarding the differential efficacy of naltrexone for reward drinkers and acamprosate for relief drinkers. The 30item version of the IDS (Annis et al, 1987) was used to identify subgroups of individuals with $\mathrm{AD}$ with distinct patterns of reward and relief drinking tendencies. We identified four latent reward/relief drinking subgroups: an all high subgroup; a reward subgroup (reward drinkers); a relief subgroup (relief drinkers); and an all low subgroup. Consistent with hypotheses, we found a significant interaction between the reward subgroup and naltrexone in the prediction of any heavy drinking during treatment. Reward drinkers who had been randomized to naltrexone had a lower likelihood of any heavy drinking during treatment (large effect size). Supplemental analyses confirmed that these findings were independent of a number of potential confounders, such as genotype (no significant allele frequency differences for the A118G polymorphism of the OPRM1 gene between the reward and relief drinking groups), and no differences on numerous other covariates that differentiated the subgroups (eg, $\mathrm{AD}$ severity, depression, stress, age, etc.). The same was true for smoking status, an important point since two recent papers reported that naltrexone may be particularly beneficial for alcohol dependent smokers. Large differences in smoking prevalences were reported in US studies (Fucito et al, 2012, reported 55\% smokers and Schacht et al, 2017, reported $39.7 \%$ ) versus our study conducted in Germany (74.1\%). We suggest that lower prevalence rates in the US indicate a more severe group of smokers that may respond better to naltrexone than samples with higher rates of smokers where naltrexone was not effective in the total sample. Moreover, to facilitate clinical application of the findings, we conducted supplementary analyses with a cutoff to identify reward drinkers based on total reward subscale score that is higher than their total relief subscale score and a total reward subscale score within the range of 12 and 31 . When using this cutoff, $31 \%$ of the sample was classified as reward drinkers and there was a large effect of naltrexone on any heavy drinking in this subgroup. Hence, when it comes to identifying patients who respond well to naltrexone, knowing whether a person's reward tendencies are stronger than relief drinking tendencies appears to be essential, in addition to knowing the absolute level of reward drinking tendencies.

One key implication from the current study is naltrexone may be a particularly effective pharmacotherapy option in reward drinkers. This finding is consistent with our understanding of the mechanisms of action of naltrexone (Littleton, 1995; Myrick et al, 2008) and with previous studies that have demonstrated that naltrexone may be particularly effective for individuals with $\mathrm{AD}$ who have frequent drinkers in their social network (Worley et al, 2015) and who have elevated pre-treatment cue reactivity in the ventral striatum (Mann et al, 2014; Schacht et al, 2017).

Importantly, construct validity analyses supported the validity of the four reward/relief subgroups identified in this study. In line with theoretical notions about reward and relief drinkers, we found that relative to the relief subgroup, the reward subgroup had lower scores on $\mathrm{AD}$ severity, depressive symptoms, and harm avoidance, and higher scores on novelty seeking. Conversely, the relief subgroup showed higher values for trait anxiety, perceived stress, and severity items.

Counter to our hypotheses, we did not find an effect of acamprosate for the relief subgroup. Prior work (Roos et al, 2017) described a positive treatment response to acamprosate among relief drinkers in the COMBINE study. The discrepancy between the findings in the current study might be explained by the length of abstinence before randomization between COMBINE (in which $66 \%$ of the sample were abstinent only 1 week or less before randomization; Anton et al, 2006) and PREDICT where participants completed inpatient medical detoxification before randomization and had been abstinent for 22 days, on average, before entering the study. This difference in abstinence days before randomization in COMBINE and PREDICT is likely to have an impact on the glutamatergic neurotransmission and the effects of acamprosate for predominantly relief drinkers. Specifically, an important role of glutamate in the pathophysiology of alcoholism has long been known (Tsai and Coyle, 1998). The glutamate hypothesis of acamprosate's mechanism of action posits that subjects in a hyperglutamatergic state are more likely to respond to a medication with anti-glutamatergic properties (Holmes et al, 2013). Glutamate brain levels both in humans and rodents are elevated in withdrawal and normalize within a few weeks of abstinence (Hermann et al, 2012). Therefore, a longer time span since the last drink in PREDICT, as compared to COMBINE, may be a disadvantage for the precision medicine hypothesis that acamprosate would work better for relief drinkers in PREDICT and may explain the disparate findings between the current study and prior work (Roos et al, 2017).

Unexpectedly, for the reward subgroup, acamprosate (relative to placebo) predicted less likelihood of any heavy drinking during treatment (medium effect size), but this effect was not significant in simple slope models or the sensitivity models controlling for compliance and additional covariates. Thus, when considering medication compliance and other covariate predictors, acamprosate did not appear to differentially benefit reward drinkers. 


\section{Limitations}

There are several limitations of the current study. The patients were treatment-seeking individuals with $\mathrm{AD}$, thus the results from the current analysis might not generalize to a non-treatment-seeking population. The overlap of the reward and relief factors is also a limitation in that the reward subgroup also endorsed some relief temptation and the relief class endorsed some reward temptation, which replicates prior work (Glöckner-Rist et al, 2013; Roos et al, 2017). It may be useful to develop subtypes that distinguish more clearly between reward and relief drinkers. Finally, patients were not randomized according to their status as a reward or relief drinker and we cannot fully exclude that an unknown factor associated with being a reward drinker predicted greater naltrexone response. Future studies should consider randomizing based on the reward subgroup selection criteria identified in the current study and evaluate whether naltrexone is more efficacious for this subgroup using this a priori definition of reward drinking.

\section{CONCLUSION AND FUTURE DIRECTIONS}

There are several ways of phenotyping to inform precision medicine. The current analysis used a person-centered, psychometric approach to identify patterns of individuals with similar responses on a relatively brief (30-item) selfreport questionnaire. The results indicate individuals with greater reward drinking tendencies than relief drinking tendencies and who have relatively high reward drinking tendencies may benefit the most from naltrexone treatment. To facilitate clinical application of the current findings, the appendix of this paper presents the items of the IDS-30 and provides instructions for how to score the IDS-30 and how to identify a person as a predominantly reward drinker.

The current analysis provides clinical support for the neurocircuitry of addiction as proposed by Koob and Volkow (2010), specifically with respect to distinct types of drinkers that may be in the 'intoxication/binge drinking' stage (ie, reward drinkers) $v s$ the 'withdrawal, negative affect' stage (ie, relief drinkers). Replication of the naltrexone by reward drinker interaction effect in a separate sample would provide greater confidence for our conclusions. Those studies could utilize the IDS-30 and the cutoff selection criteria used in this study for identifying reward drinkers. Further, examining the association between the reward and relief drinking and imaging of cue reactivity and stress reactivity could further substantiate the reward/relief phenotypes.

\section{FUNDING AND DISCLOSURE}

KM has received honoraria from Lundbeck and Pfizer. The remaining authors declare no conflict of interest.

\section{ACKNOWLEDGMENTS}

We thank the members of the Predict Study Group (Mann et al, 2013) for their support and also for the Co-PIs of the different centers: Michael Smolka, Falk Kiefer, Stefan Wellek, Rosemarie Krämer, Silke Merkel, and Iris Reinhard (Mannheim); Michael Berner, Katrin Frick, and Horst Gann (Freiburg);
Johannes Schröder and Johannes Pantel (Heidelberg); Anil Batra (Tübingen); Norbert Wodarz and Monika Johann (Regensburg); Hans Hagenbuch (Emmendingen); and Barbara Richter and Matthias Kluge (Wiesloch). We are also grateful to Ron Mucha, PhD for his initial advice concerning IDS and subgroup analyses. Angelika Heimann provided excellent assistance in the publication process.

This research was supported by the Federal Government of Germany (01EB0110) and the United States National Institute on Alcohol Abuse and Alcoholism (T32 AA018108, R01 AA022328, and R01 AA025539). Medication was donated by Bristol-Myers Squibb and MERCK Serono. KW and CR were funded by the National Institute on Alcohol Abuse and Alcoholism. SH, HN, and TL were funded by the Central Institute of Mental Health, Mannheim. KM and KW are members of the American Society of Clinical Psychopharmacology's Alcohol Clinical Trials Initiative (ACTIVE Group, Anton et al, 2012).

\section{REFERENCES}

Annis HM, Graham JM, Davis CS (1987). Inventory of Drinking Situations: User's Guide. Addiction Research Foundation: Toronto, ON, Canada.

Anton RF, O'Malley SS, Ciraulo DA, Cisler RA, Couper D, Donovan $\mathrm{DM}$ et al (2006). Combined pharmacotherapies and behavioral interventions for alcohol dependence: the COMBINE study: a randomized controlled trial. JAMA 295: 2003-2017.

Anton RF, Litten RZ, Falk DE, Palumbo JM, Bartus RT, Robinson RL et al (2012). The Alcohol Clinical Trials Initiative (ACTIVE): purpose and goals for assessing important and salient issues for medications development in alcohol use disorders. Neuropsychopharmacology 37: 402-411.

Beck AT, Steer RA, Brown GK (1996). Manual for the Beck Depression Inventory-II. Psychological Corporation: San Antonio, TX, USA.

Cloninger CR, Svrakic DM, Przybeck TR (1993). A psychobiological model of temperament and character. Arch Gen Psychiatry 50: 975-990.

Cohen S, Kamarck T, Mermelstein R (1983). A global measure of perceived stress. J Health Soc Behav 24: 385-396.

Fucito LM, Park A, Gulliver SB, Mattson ME, Gueorguieva RV, O'Malley SS (2012). Cigarette smoking predicts differential benefit from naltrexone for alcohol dependence. Biol Psychiatry 72: $832-838$.

Glöckner-Rist A, Lémenager T, Mann K,PREDICT Study Research Group (2013). Reward and relief craving tendencies in patients with alcohol use disorders: results from the PREDICT study. Addict Behav 38: 1532-1540.

Heatherton TF, Kozlowski LT, Frecker RC, Fagerström KO (1991). The Fagerström test for nicotine dependence: a revision of the Fagerström Tolerance Questionnaire. Addiction 86: 1119-1127.

Heilig M, Leggio L (2016). What the alcohol doctor ordered from the neuroscientist. Prog Brain Res 224: 401-418.

Heilig M, Thorsell A, Sommer WH, Hansson AC, Ramchandani VA, George DT et al (2010). Translating the neuroscience of alcoholism into clinical treatments: from blocking the buzz to curing the blues. Neurosci Biobehav Rev 35: 334-344.

Heinz A, Löber S, Georgi A, Wrase J, Hermann D, Rey E-R et al (2003). Reward craving and withdrawal relief craving: assessment of different motivational pathways to alcohol intake. Alcohol Alcohol 38: 35-39.

Hermann D, Weber-Fahr W, Sartorius A, Hoerst M, Frischknecht U, Tunc-Skarka N et al (2012). Translational magnetic resonance spectroscopy reveals excessive central glutamate levels during 
alcohol withdrawal in humans and rats. Biol Psychiatry 71: 1015-1021.

Hillmer AT, Mason GF, Fucito LM, O’Malley SS, Cosgrove KP (2015). How imaging glutamate, $\gamma$-aminobutyric acid, and dopamine can inform the clinical treatment of alcohol dependence and withdrawal. Alcohol Clin Exp Res 39: 2268-2282.

Holmes A, Spanagel R, Krystal JH (2013). Glutamatergic targets for new alcohol medications. Psychopharmacology 229: 539-554.

Institute of Medicine (1990). Broadening the Base of Treatment for Alcohol Problems. National Academies Press: Washington, DC, USA.

Jonas DE, Amick HR, Feltner C, Bobashev G, Thomas K, Wines R et al (2014). Pharmacotherapy for adults with alcohol use disorders in outpatient settings: a systematic review and metaanalysis. JAMA 311: 1889-1900.

Koob GF, Mason BJ (2016). Existing and future drugs for the treatment of the dark side of addiction. Annu Rev Pharmacol Toxicol 56: 299-322.

Koob GF, Volkow ND (2010). Neurocircuitry of addiction. Neuropsychopharmacology 35: 217-238.

Kranzler HR, Armeli S, Covault J, Tennen H (2013). Variation in OPRM1 moderates the effect of desire to drink on subsequent drinking and its attenuation by naltrexone treatment. Addict Biol 18: 193-201.

Kranzler HR, McKay JR (2012). Personalized treatment of alcohol dependence. Curr Psychiatry Rep 14: 486-493.

Litten RZ, Castle I-JP, Falk D, Ryan M, Fertig J, Chen CM et al (2013). The placebo effect in clinical trials for alcohol dependence: an exploratory analysis of 51 naltrexone and acamprosate studies. Alcohol Clin Exp Res 37: 2128-2137.

Litten RZ, Ryan ML, Falk DE, Reilly M, Fertig JB, Koob GF (2015). Heterogeneity of alcohol use disorder: understanding mechanisms to advance personalized treatment. Alcohol Clin Exp Res 39: $579-584$.

Littleton J (1995). Acamprosate in alcohol dependence: how does it work? Addiction 90: 1179-1188.

Magill M, Ray LA (2009). Cognitive-behavioral treatment with adult alcohol and illicit drug users: a meta-analysis of randomized controlled trials. J Stud Alcohol Drugs 70: 516-527.

Mann K, Kiefer F, Smolka M, Gann H, Wellek S, Heinz A et al (2009). Searching for responders to acamprosate and naltrexone in alcoholism treatment: rationale and design of the Predict Study. Alcohol Clin Exp Res 33: 674-683.

Mann K, Leménager T, Hoffmann S, Reinhard I, Hermann D, Batra A et al (2013). Results of a double-blind, placebo-controlled pharmacotherapy trial in alcoholism conducted in Germany and comparison with the US COMBINE study. Addict Biol 18: 937-946.

Mann K, Vollstädt-Klein S, Reinhard I, Leménager T, Fauth-Bühler M, Hermann D et al (2014). Predicting naltrexone response in alcohol-dependent patients: the contribution of functional magnetic resonance imaging. Alcohol Clin Exp Res 38: 2754-2762.

Miller WR (1996). Form 90: A Structured Assessment Interview for Drinking and Related Behaviors. National Institute on Alcohol Abuse and Alcoholism: Bethesda, MD, USA.

Muthén LK, Muthén BO (2012). Mplus users guide (Version 7). Muthén \& Muthén: Los Angeles, CA, USA.

Myrick H, Anton RF, Li X, Henderson S, Randall PK, Voronin K (2008). Effect of naltrexone and ondansetron on alcohol cueinduced activation of the ventral striatum in alcoholdependent people. Arch Gen Psychiatry 65: 466-475.

Nylund KL, Asparouhov T, Muthén BO (2007). Deciding on the number of classes in latent class analysis and growth mixture modeling: a Monte Carlo simulation study. Struct Equ Model 14: 535-569.
O’Malley SS, Jaffe AJ, Chang G, Rode S, Schottenfeld R (1996). Sixmonth follow-up of naltrexone and psychotherapy for alcohol dependence. Arch Gen Psychiatry 53: 217-224.

Oslin DW, Leong SH, Lynch KG, Berrettini W, O’Brien CP, Gordon AJ et al (2015). Naltrexone vs Placebo for the Treatment of Alcohol Dependence. JAMA Psychiatry 72: 430.

Pettinati HM, Weiss RD, Miller WR, Donovan DM, Ernst D, Rounsaville BJ (2004). COMBINE Monograph Series, Volume 2. Medical Management Treatment Manual: A Clinical Research Guide for Medically Trained Clinicians Providing Pharmacotherapy as Part of the Treatment for Alcohol Dependence. National Institute on Alcohol Abuse and Alcoholism: Bethesda, MD, USA.

Roos CR, Mann K, Witkiewitz K (2017). Reward and relief dimensions of temptation to drink: construct validity and role in predicting differential benefit from acamprosate and naltrexone. Addict Biol 22: 1528-1539.

Schacht JP, Anton RF, Myrick H (2013). Functional neuroimaging studies of alcohol cue reactivity: a quantitative meta-analysis and systematic review. Addict Biol 18: 121-133.

Schacht JP, Randall PK, Latham PK, Voronin KE, Book SW, Myrick $\mathrm{H}$ et al (2017). Predictors of naltrexone response in a randomized trial: reward-related brain activation, OPRM1 genotype, and smoking status. Neuropsychopharmacology 42: 2640-2653.

Sinha R, Li CSR (2007). Imaging stress- and cue-induced drug and alcohol craving: association with relapse and clinical implications. Drug Alcohol Rev 26: 25-31.

Skinner HA, Horn JL (1984). Alcohol Dependence Scale (ADS) User's Guide. Addiction Research Foundation: Toronto, ON, Canada.

Spanagel R, Durstewitz D, Hansson A, Heinz A, Kiefer F, Köhr G et al (2013). A systems medicine research approach for studying alcohol addiction. Addict Biol 18: 883-896.

Spanagel R, Kiefer F (2008). Drugs for relapse prevention of alcoholism: ten years of progress. Trends Pharmacol Sci 29: 109-115.

Spanagel R, Weiss F (1999). The dopamine hypothesis of reward: past and current status. Trends Neurosci 22: 521-527.

Spielberger CD, Gorusch RL, Lushene R, Vagg PR, Jacobs GA (1983). Manual for the State-Trait Anxiety Inventory. Consulting Psychologists Press: Palo Alto, CA, USA.

Treutlein J, Cichon S, Ridinger M, Wodarz N, Soyka M, Zill P et al (2009). Genome-wide association study of alcohol dependence. Arch Gen Psychiatry 66: 773.

Tsai G, Coyle JT (1998). The role of glutamatergic neurotransmission in the pathophysiology of alcoholism. Annu Rev Med 49: $173-184$.

van den Brink W, Aubin H-J, Bladström A, Torup L, Gual A, Mann $\mathrm{K}$ (2013). Efficacy of as-needed nalmefene in alcohol-dependent patients with at least a high drinking risk level: results from a subgroup analysis of two randomized controlled 6-month studies. Alcohol Alcohol 48: 570-578.

Verheul R, Brink W, van den, Geerlings P (1999). A three-pathway psychobiological model of craving for alcohol. Alcohol Alcohol 34: 197-222.

Victorio-Estrada A, Mucha RF, Stephan ER (1996). Excessive drinking situations in German alcoholics: replication of a threefactor model used for North Americans. Drug Alcohol Depend 41: 75-79.

World Health Organization (2009). Global Health Risks: Mortality and Burden of Disease Attributable to Selected Major Risks. World Health Organization: Geneva, Switzerland.

Worley MJ, Witkiewitz K, Brown SA, Kivlahan DR, Longabaugh R (2015). Social network moderators of naltrexone and behavioral treatment effects on heavy drinking in the COMBINE study. Alcohol Clin Exp Res 39: 93-100. 


\section{Appendix}

Appendix Inventory of Drinking Situations ( Annis et al, 1987)-30 item Version (IDS-30)

Listed below are situations in which some people drink heavily. Read each item carefully, and answer in terms of your own heavy drinking in the past year.

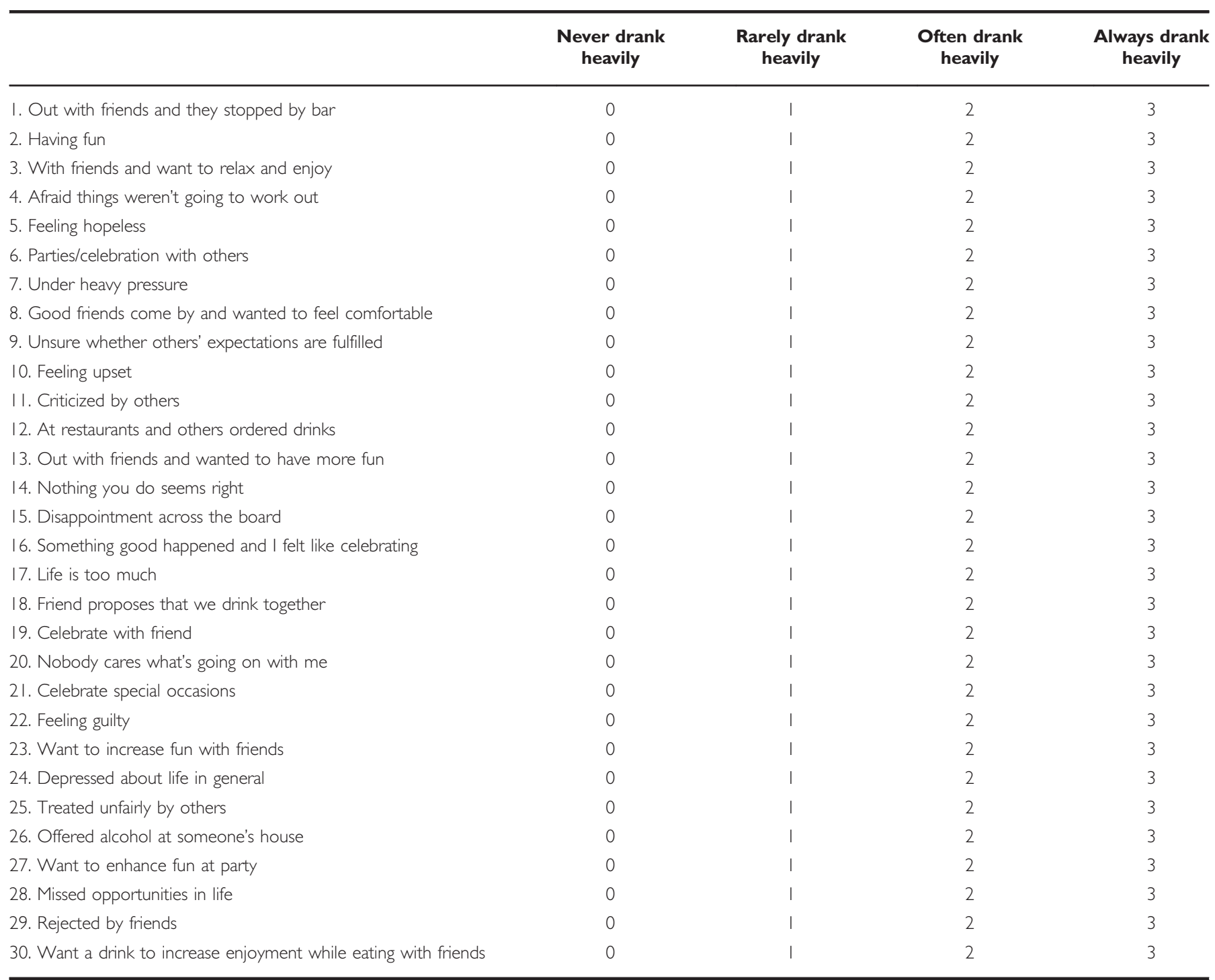

Instructions for using the IDS-30 to identify reward drinkers

1. Calculate reward drinking score by summing items $1,2,3,6,8,12,13,16,18,19,21,23,26,27$, and 30 . Reward subscale score

2. Calculate relief drinking scores by summing items $4,5,7,9,10,11,14,15,17,20,22,24,25,28$, and 29 . Relief subscale score

IF... a person meets BOTH of the following two criteria:

1. The reward subscale score is higher than the relief subscale score.

2. The reward subscale score is within the range of 12 and 31 .

THEN... this person can be classified as a reward drinker and is a good candidate for naltrexone treatment.

Supplementary Information accompanies the paper on the Neuropsychopharmacology website (http://www.nature.com/npp) 